\title{
Prevalence and determinants of modern contraceptive utilization among rural lactating mothers: Findings from the primary health care Project in two northwest Ethiopian Districts
}

Terefe Derso ( $\nabla$ dersotere@gmail.com )

University of Gondar

Gashaw Andargie Biks

University of Gondar

Mezgebu Yitayal

University of Gondar

Tadesse Awoke Ayele

University of Gondar

Kassahun Alemu

University of Gondar

Getu Debalkie

University of Gondar

Hailab Fekadu

University of Gondar

Endalkachew Dellie

University of Gondar

Telake Azale

University of Gondar

Bisrat Misganaw

University of Gondar

Adane Kebede

University of Gondar

Destaw Fetene

University of Gondar

Tsegaye G/medhin

University of Gondar

Asmamaw Atnafu

University of Gondar

Research article

Keywords: Contraceptive utilization, Postnatal care, Child immunization

Posted Date: October 4th, 2019 
DOI: https://doi.org/10.21203/rs.2.15782/v1

License: (c) (i) This work is licensed under a Creative Commons Attribution 4.0 International License. Read Full License

Version of Record: A version of this preprint was published at BMC Women's Health on April 3rd, 2020. See the published version at https://doi.org/10.1186/s12905-020-00933-7. 


\section{Abstract}

Background Contraceptives utilization is a guarantee to avert unwanted pregnancy. However, in Ethiopia more than half of the rural women have shorter birth intervals. Also of all births in their pregnancies, $17 \%$ and $8 \%$ were mistimed (wanted at a later date) and unwanted births, respectively. Therefore, this paper investigates modern contraceptive utilization and predictors among rural lactating women.

Methods community cross-sectional study was conducted from May 01 to June 29, 2019 in two (Dabat and Gondar zuria district) northwest Ethiopian Districts. A total of 603 lactating mothers were included in this study. Data was collected through face to face interview using a structured questionnaire. Bivariate and multivariate logistic regression analyses were done to identify the independent predictors of modern contraceptive utilization.

Results The overall prevalence of modern contraceptive (MC) utilization rate was 45.8\% [95\% Cl: $38.01,53.59]$. The contraceptive method mix was dominated by Depo-Provera (39.8\%) and followed by implants (4.8\%). The odds of modern utilization of contraceptive were 5.58 times higher among with children with fully immunization [AOR=5.58, $95 \% \mathrm{Cl}: 3.45,9.01]$ compared to mothers with children with incomplete or not vaccinated. Mothers who received antenatal care $[A O R=1.743,95 \% \mathrm{Cl}: 1.13,4.43]$ and postnatal care[AOR=2.02, 95\%Cl: 1.24, 2.91) were 1.74 and 2.02 folds more likely utilize modern contraceptive than mothers who did not received antenatal care and postnatal care, respectively.

Conclusion in the study area, low prevalence of modern contraceptive utilization is documented compared to the planed national figure. In the region child immunization services is one of a promising platform to reach lactating mothers with modern contraceptive utilization. Also our findings suggest antenatal care and postnatal care visit are the other key determinants for modern contraceptive utilization. According to our findings in a low-resource setting like Ethiopia, the health system approach to improved ANC, postnatal care and child immunization services should be intensified with more effective advice on modern contraceptive utilization to reduce unwanted pregnancy.

\section{Background}

The health benefits of Contraceptive use: preventing unplanned pregnancies [1] and potential to avert maternal and child morbidity by allowing couples to space their pregnancies more than 2 years apart $[2,3,4]$. In spite of, more than half of lactating women are at high risk for unwanted or unplanned pregnancy immediately after birth [5], the postpartum period, a time of care for themselves and their newborns is an important opportunities of contact with the health system [6]. Accordingly, postpartum modern contraceptive has the potential to reduce $71 \%$ of unwanted pregnancies: abolishing 53 million unintended pregnancy, 22 million fewer unplanned births, 25 million fewer induced abortion and 7 million fewer miscarriages [7]. Also, longer birth intervals or contraceptive use can avert about 1 in 5 deaths in children of 1 to 4 years of age [8] and 44.3\% of maternal deaths [9].

Since 1993 Ethiopia is implementing a clear population policy [10] and currently, one of the major strategies of the policy is family planning program and plan to rises contraceptive prevalence to $55 \%$ by 2020 through free of charge providing in both governmental and non-governmental health facilities $(11,12)$. So far, in Ethiopia, Low in modern contraceptive prevalence (35\%) exist with significant regionally variations: Somali (1\%), Affar (12\%) and Amhara (47). Consequently, of all births in their pregnancies, $17 \%$ and $8 \%$ were mistimed (wanted at a later date) and unwanted births, respectively at all [13]. 
Contraceptive uptake has been positively associated with a range of both health and non-health related outcomes. Factors that influence contraceptive practice are multifaceted and complex. Previous studies showed that socio-cultural, health and socio-economic characteristics were main significant determinants of modern contraceptive utilization. High contraceptive utilization is documented in women who are literate [13, 14], old age [14], living in rural residences [13], exposure to media and richest wealth status [15] and large number of living children ( $>$ five children) [16, 17]. Health care utilizations during pregnancy and after delivery: antenatal; and postnatal care [18, 19] and child immunization [20] also increase the likelihood of modern contraceptive utilization.

Mothers wish to space their births sufficiently far apart that even when their child is a toddler, they prefer to delay pregnancy [21]. However, in Ethiopia more than half of the rural women have shorter birth intervals and consequently, high maternal mortality rate is documented [13]. Also subsequent conceptions and births negatively affect a mother's decision to continue breast-feeding a child [22]. Some local studies have been focused to assess the contraceptive utilization status of women generally $[16,17$,$] even only two local studies targeted to urban lactating women [23,24]$. Thus, the lactating (postnatal) period is a window of opportunity to improve contraceptive utilization. Investigating the prevalence and predictors of modern contraceptive utilization in rural area is of a vital importance to design strategies to address the problem. Therefore, this paper investigates modern contraceptive utilization and predictors among rural lactating women.

\section{Methods}

Study setting and design

A community-based cross-sectional study was conducted from May 01 to June 29, 2019 in the two (Dabat and Gondar zuria district) northwest Ethiopian Districts. Dabat and Gondar zuria districts are the two neighboring districts among 23 districts in north Gondar Amhara region, which consist of 30 and 38 kebeles(smallest administrative unit in Ethiopia), respectively. The two districts found in different ecological zones (high land, middle land, and low land), and has 145,509 inhabitants (Dabat district) and 231,324 inhabitants (Gondar district). For their livelihood the residents largely depend on subsistence farming.

Study population and sampling procedure

Lactating mothers who lived in Dabat and Gondar zuria districts for at least six months were included in the study. Initially, the study was aimed to assess accessibility and availability of primary healthcare services at the community level, in Dabat and Gondar zuria districts, Northwest Ethiopia. Of the total kebeles, eight kebeles in Dabat district and ten kebeles in Gondar zuria district were selected using lottery method. For households with multiple children fulfilling the inclusion criteria, a child was selected using lottery method.

As part of the original survey, this particular study utilized the data extracted from the original survey database. Accordingly, only lactating mothers were considered to investigate the magnitude of modern contraceptive utilization and predictors. Sample size was calculated using Epi-info version 3.7 by considering the following assumptions: $48.4 \%$ as the prevalence of modern contraceptive utilization in Gondar Town [23], 95\% level of confidence and 5\% margin of error. A design effect of 1.5 and $10 \%$ non-response rate were also anticipated which gives a final sample size of 631 . 
Data collection tools and procedure

A structured pre-tested questionnaire was designed to capture socio-demographic characteristics and primary health care utilization (immunization, family planning, health education, maternal health service and physical access to health services). The questionnaire was first prepared in English language, and later translated to Amharic (the native language of the study area). Back translation to English was done to compare the consistency, and amendments were made accordingly. Fifteen data collectors and three field supervisors were recruited for collect the data. the data collectors and supervisors were trained for two days.

Study variables and data analysis

To ascertain the outcome variable, modern utilization of contraceptive, women were asked if they were currently using a method of contraception and what method they were using. The outcome was coded 1 if they were using a modern method (pill, Intra uterine contraceptive device (IUD), injections, condom, male or female sterilization, implant, or diaphragm/foam/jelly) and 0 if they were using a traditional method, folkloric method, or were not currently using a method. Epi-data version 3.1 was used for data entry and data were exported to SPSS version 21 for analysis. Descriptive statistics were computed. Binary Logistic regression model was used to identify the relationship between dependent and independent variables. Those independent variables which were significant in bivariate analysis ( $p$-value < 0.0.2) were entered into the multivariable analysis. In the binary logistic regression model, backward- stepwise multivariate analysis was used to elicit associated factors of modern utilization of contraceptive. In the final model, a significant association was declared at a pvalue less than 0.05 and finally, the results were presented in texts and tables with adjusted odds ratio (AOR) and the corresponding 95\% confidence interval.

\section{Results}

\section{Socio-demographic and health related characteristics of study participants}

A total of 603 mothers (with a response rate of 95.56\%) were participated in the study. The great majority of mothers were housewife (94.7\%). Only nearly one-fourth of mothers had formal education (22.1\%) and in the age ranges of 36-49 years (22.9\%) (Table 1).

Table 1: Socio-demographic and health related characteristics of study participants in the rural population of northwest Ethiopia 


\begin{tabular}{|l|l|l|}
\hline Characteristics & Frequency & Percent \\
\hline Age of mothers(in years) & & \\
\hline $18-24$ & 102 & 16.9 \\
\hline $25-35$ & 363 & 60.2 \\
\hline $36-49$ & 138 & 22.9 \\
\hline Maternal educational status & & \\
\hline Unable to read and write & 400 & 66.3 \\
\hline Able to read and write without formal education & 70 & 11.6 \\
\hline Formal education & 137 & 22.1 \\
\hline Husband's educational status & & \\
\hline Unable to read and write & 344 & 57.0 \\
\hline Able to read and write without formal education & 133 & 22.1 \\
\hline First cycle(1-4 grade) & 41 & 6.8 \\
\hline Second cycle(5-8 grade) & 50 & 8.3 \\
\hline Secondary school(9-12 grade) & 26 & 4.3 \\
\hline Certificate and above & 9 & 1.5 \\
\hline Occupation of mothers & & \\
\hline House wife & 571 & 94.7 \\
\hline Outdoor worker & 32 & 5.3 \\
\hline Number of children & & \\
\hline 1-4 & 422 & 70.0 \\
\hline Above 4 children & 181 & 30.0 \\
\hline
\end{tabular}

\section{Health related characteristics of study participants}

Of all participants, 95.2\% have had antenatal care (ANC) visits. Also the vast majority (70.0\%) of mothers gave birth in health institutions (Table 2).

Table 2: Health related characteristics of study participants in the rural population of northwest Ethiopia 


\begin{tabular}{|l|l|l|}
\hline Characteristics & Frequency & Percent \\
\hline Travel time to the nearest health center(HC) & & \\
\hline 30 or below minutes & 146 & 24.2 \\
\hline Above 30 minutes & 457 & 75.8 \\
\hline ANC visit during your last pregnancy & & \\
\hline Yes & 574 & 95.2 \\
\hline No & 29 & 4.8 \\
\hline Place of last delivery & & \\
\hline Home & 181 & 30.0 \\
\hline Health institution & 422 & 70.0 \\
\hline Postnatal care in the current delivery & & \\
\hline Yes & 397 & 65.8 \\
\hline No & 206 & 34.2 \\
\hline Place of postnatal care & & \\
\hline Hospital & 22 & 3.6 \\
\hline Health Center & 267 & 44.3 \\
\hline Health Post & 108 & 17.9 \\
\hline Child immunization status & & \\
\hline Fully vaccinated & 464 & 76.9 \\
\hline Partially/not vaccinated & 139 & 23.1 \\
\hline
\end{tabular}

Prevalence of modern contraceptive utilization

The prevalence of modern contraceptive (MC) utilization was 45.8\% [95\% CI: 38.01, 53.59]. The contraceptive method mix was dominated by Depo-Provera (39.8\%) and followed by implants (4.8\%). However, very few mothers $(1.2 \%$ and $0.2 \%$, respectively) were utilized pills and Intra uterine contraceptive device.

\section{Factors associated with modern contraceptive utilization}

In the bivariable analysis age of mothers, number of children, place of last delivery, travel time to the nearest health center, antenatal care visit, postnatal care and child immunization status were found associated with a p-value of less than 0.2. However, the result of the multivariable analysis revealed with a p-value of less than 0.05 that the odds of modern utilization of contraceptive were 5.58 times higher among with children with fully immunization [AOR=5.58, 95\% CI: 3.45, 9.01] compared to mothers with children with incomplete or not vaccinated. Mothers who received antenatal care $[\mathrm{AOR}=1.743,95 \% \mathrm{CI}: 1.13,4.43]$ and postnatal care[AOR=2.02, 95\%CI: 1.24, 2.91) were 1.74 and 2.02 folds more utilize modern contraceptive than mothers who did not received antenatal care and postnatal care, respectively (Table 3).

Table 3: Factors associated with modern utilization of contraceptive in the rural population of northwest, Ethiopia 


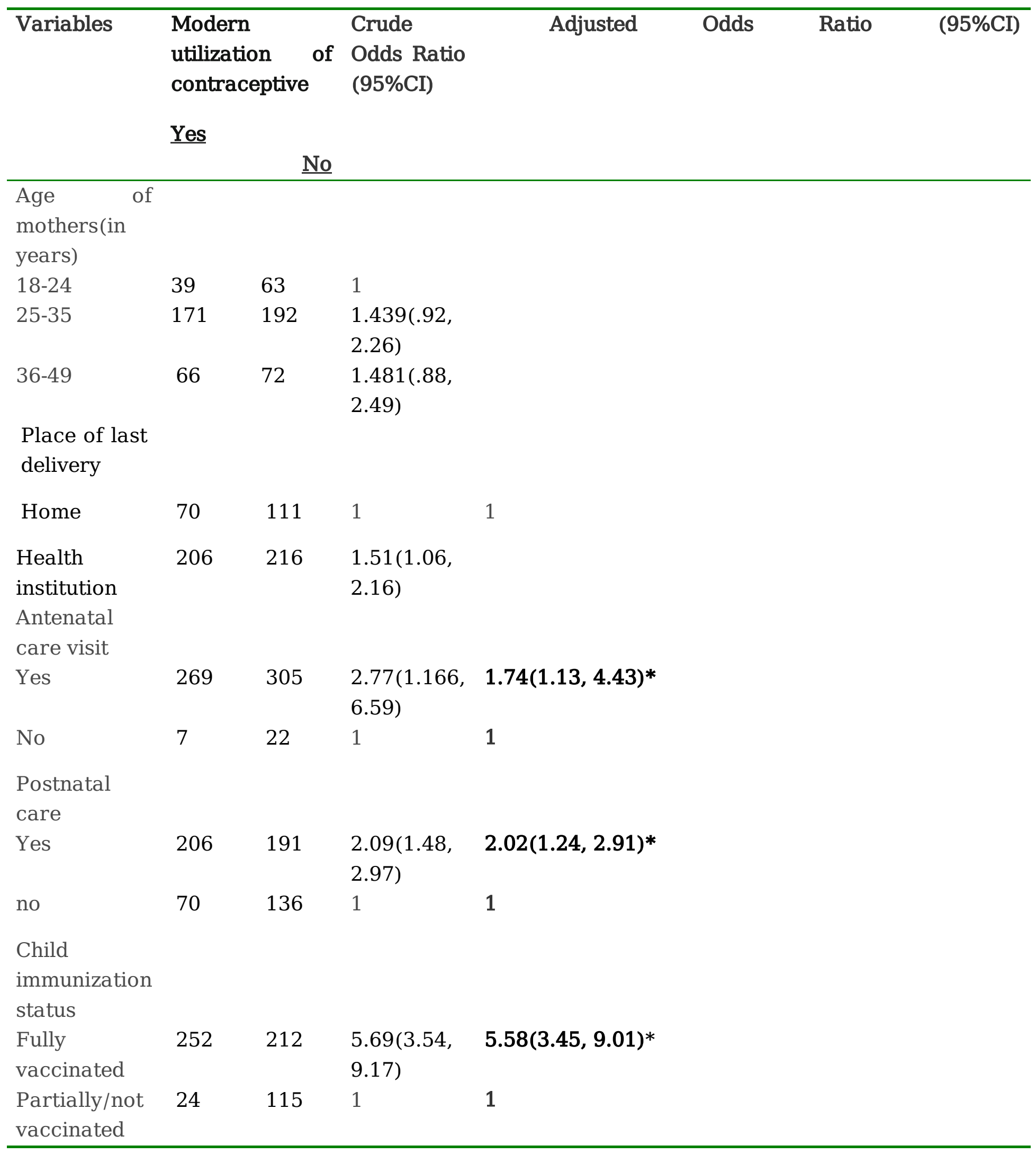

\section{Discussion}

Contraceptives utilization is a guarantee to avert unwanted pregnancy [1]. This community based cross-sectional study revealed that overall prevalence of modern contraceptive utilization was $45.8 \%$ 
[95\% CI: 38.01, 53.59]; antenatal care, postnatal care and child immunization are the predictors of modern utilization of contraceptive. In this finding the magnitude of modern contraceptive utilization is consistent with previous studies reported from; North Shoa Zone (46.9\%) [16], Gondar city (48.4\%)[23] and Adigrat town, Ethiopia(51.3\%)[25]. Also this finding is comparable with the finding documented in the two southwestern Nigerian states (43.9\%)[26].

However, the magnitude of modern contraceptive utilization is lower than the finding in Malawi where three-fourth (75\%) of the lactating women were utilizing contraceptives [27]. This difference might be due to variation in awareness of the people, availability of the contraceptive methods and the difference in study settings to access the service. also the high prevalence in Malawi compared to the current study might be due to the education level, in the current study more than three-fourth (77.9\%) of the study participants were illiterate but in the former study, Malawi, great majority (90.2\%) of study participants had primary and above education level. This could be due to education enables/supports/ mothers to improve knowledge and awareness about the benefits of family planning and this enhance mothers' modern contraceptive utilization. This implies that, educated women have greater ability to stick to health care inputs such as modern contraceptive utilization which offer better for spacing of birth. Comparable to the previous studies the dominant modern contraceptive method used was Depo-Provera [13, 16, 17]. This might be due to the ease of the procedure to use [25].

Mothers who received antenatal care were $\mathbf{1 . 7 4}$ folds more likely utilize modern contraceptive than mothers who did not received antenatal care. This higher odds of modern contraceptive utilization is supported with findings reported from Ethiopia [19] and Nigeria [18]. The plausible reason might be during antenatal care follow up the mothers get counseling on birth spacing and family planning. This implies that antenatal care is important to reach women before they are at risk for an unintended pregnancy with information about return of fertility, their options to space or limit future pregnancies, and the benefits to their own and their newborn's health of doing so. It is further recommended that women should be counseled on birth spacing and family planning during ANC contacts.

In the multivariate analysis, postnatal care is another significant variable, mothers who received postnatal care 2.02 times more likely utilize modern contraceptive than mothers who did not received postnatal care. This finding is in line with other previous findings in Africa [19, 18]. This might be due to postnatal care enable health workers to counsel women on the importance of child spacing. This implies that postnatal care should be prioritized with more effective advice on family planning to reduce unintended births.

The uncommon finding, this study showed that likelihoods of modern utilization of contraceptive were 5.58 times higher among lactating mothers with children fully immunization compared to mothers with children with incomplete or not vaccinated. In fact the recommended vaccination schedule for children allows for multiple health care contacts with infants and their mothers during the first year of life [20]. This routine immunization services offer important opportunities to discuss family planning with the women and is an opportunity to reach many postpartum women during group education talks about family planning. In Ethiopia immunization contacts occur regularly according to the country schedule, such as at 6 weeks, 10 weeks, 14 weeks, 9 months and 15 months [28]. Thus, child immunization services are a Promising Platform to reach lactating mothers with modern contraceptive utilization [29]. Finally, advocacy efforts can promote positive partnerships 
between child health and family planning programmes as a 'win-win' for the health of both the mother and the infant.

Limitation: Findings of this study present important insight about modern contraceptive utilization and its predictors among lactating mothers, in Dabat and Gondar zuria districs where there is scarcity of literature. However, the study has limitation, as an illustration the cross sectional nature of the study limits measuring the cause and effect relationship between the outcome and the potential predictors.

\section{Declarations}

List of abbreviations

AOR: Adjusted Odds Ratio; ANC: Antenatal Care; COR: Crude Odds Ratio, CI: Confidence Interval; IUD; Intra uterine contraceptive device: PNC: Postnatal care; SPSS: Statistical Package for Social Science; WHO: World Health Organization

Ethics approval and consent to participate

Ethical approval was obtained from Institutional Review Board of University of Gondar. Informed consent was also obtained from parents or legal guardians. The confidentiality of information was guaranteed by using code numbers rather than personal identifiers and by keeping the data locked. Participants were told to decline at any time if they felt uncomfortable, even after the interview was started.

Consent for publication

Not applicable

Availability of data and materials

We confirm that all data underlying the findings would be fully available without restriction if the manuscript is published.

Competing interest

Authors declare that they have no conflict of interest

Funding

This study was funded by the University of Gondar. The views presented in the article are of the author and not necessarily express the views of the funding organization. The University of Gondar was not involved in the design of the study, data collection, analysis and interpretation.

Authors contributions

AA TD GAB MY TAA conceived the study, developed the tool, coordinated data collection, and carried out the statistical analysis and drafted the manuscript. AA KA GD HF TA BM AK DF TG AA ED conceived the study, participated in the statistical analysis, and drafted the manuscript. TD AA conceived the study and reviewed the drafted manuscript. All authors read and approved the final manuscript. Acknowledgements

We would like to thank the mothers for their willingness to participate in the study. Our appreciation will also go to the University of Gondar for their financial and material support.

\section{References}

1. Sedgh G, Singh S, Hussain R. Intended and unintended pregnancies worldwide in 2012 and recent trends. Studies in family planning. 2014 Sep;45(3):301-14. 
2. DaVanzo J, Hale L, Razzaque A, Rahman M. The effects of pregnancy spacing on infant and child mortality in Matlab, Bangladesh: how they vary by the type of pregnancy outcome that began the interval. Population studies. 2008 Jul 1;62(2):131-54.

3. Chen, M.J., Kim, C.R., Whitehouse, K.C., Berry-Bibee, E. and Gaffield, M.E., 2017. Development, updates, and future directions of the World Health Organization selected practice recommendations for contraceptive use. International journal of gynaecology and obstetrics: the official organ of the International Federation of Gynaecology and Obstetrics, 136(2), p.113.

4. Singh S, Bankole A, Darroch JE. The impact of contraceptive use and abortion on fertility in sub-Saharan Africa: estimates for 2003-2014. Population and development review. 2017 May;43(Suppl 1):141.

5. ohannes S, Wondafrash M, Abera M, Girma E. Duration and determinants of birth interval among women of child bearing age in Southern Ethiopia. BMC pregnancy and childbirth. 2011 Dec;11(1):38.

6. Sines E, Syed U, Wall S, Worley H. Postnatal care: A critical opportunity to save mothers and newborns. Policy Perspectives on Newborn Health. 2007 Jan:1-7.

7. World Health Organization. Packages of interventions for family planning, safe abortion care, maternal, newborn and child health. WHO; 2010.

8. CSA I. International: Ethiopia Demographic and Health Survey 2011. Addis Ababa, Ethiopia and Calverton, Maryland, USA: Central Statistical Agency and ICF International.2012.

9. Ahmed S, Li Q, Liu L, Tsui AO. Maternal deaths averted by contraceptive use: an analysis of 172 countries. The Lancet. 2012 Jul 14;380(9837):111-25.

10. Federal Democratic Republic of Ethiopia The National Population Policy of Ethiopia; 1993

11. Federal Democratic Republic of Ethiopia, Ministry of Health. National Guideline for Family Planning Services in Ethiopia; 2011,

12. Federal Ministry of Health; Costed Implementation Plan for Family Planning in Ethiopia 2015-2020; 2016

13. CSA . International: Ethiopia Demographic and Health Survey 2016. Addis Ababa, Ethiopia and Calverton, Maryland, USA: Central Statistical Agency and ICF International.2016.

14. Gebremariam A, Gebremariam H. Contraceptive use among lactating women in Ganta-Afeshum District, Eastern Tigray, Northern Ethiopia, 2015: a cross sectional study. BMC pregnancy and childbirth. 2017 Dec;17(1):421.

15. Rutaremwa G, Kabagenyi A, Wandera SO, Jhamba T, Akiror E, Nviiri HL. Predictors of modern contraceptive use during the postpartum period among women in Uganda: a population-based cross sectional study. BMC public health. 2015 Dec;15(1):262.

16. Mohammed A, Woldeyohannes D, Feleke A, Megabiaw B. Determinants of modern contraceptive utilization among married women of reproductive age group in North Shoa Zone, Amhara Region, Ethiopia. Reproductive health. 2014 Dec;11(1):13.

17. Alemayehu GA, Fekadu A, Yitayal M, Kebede Y, Abebe SM, Ayele TA, Gizaw Z, Wubeshet M, Muchie KF, Gelagay AA, Azmeraw T. Prevalence and determinants of contraceptive utilization among married women at Dabat Health and Demographic Surveillance System site, northwest Ethiopia. BMC women's health. 2018 Dec;18(1):118.

18. Ajayi Al, Adeniyi OV, Akpan W. Maternal health care visits as predictors of contraceptive use among childbearing women in a medically underserved state in Nigeria. Journal of Health, Population and Nutrition. 2018 Dec;37(1):19.

19. Teka TT, Feyissa TR, Melka AS, Bobo FT. Role of antenatal and postnatal care in contraceptive use during postpartum period in western Ethiopia: a cross sectional study. BMC research notes. 2018 Dec;11(1):581. 
20. Wallace A, Dietz V, Cairns KL. Integration of immunization services with other health interventions in the developing world: what works and why? Systematic literature review. Tropical Medicine \& International Health. 2009 Jan;14(1):119.

21. Greenhalgh S, Bongaarts J. Fertility policy in China: future options. Science. 1987 Mar 6;235(4793):1167-72.

22. Jayachandran S. Does contraceptive use always reduce breast-feeding?. Demography. 2014 Jun 1;51(3):917-37.

23. Abera Y, Mengesha ZB, Tessema GA. Postpartum contraceptive use in Gondar town, Northwest Ethiopia: a community based cross-sectional study. BMC women's health. 2015 Dec;15(1):19.

24. Taye EB, Mekonen DG, Debele TZ. Prevalence of post partum modern family planning utilization and associated factors among postpartum mothers in Debre Tabor town, North West Ethiopia, 2018. BMC research notes. 2019 Dec;12(1):430.

25. Gebremariam A, Addissie A. Intention to use long acting and permanent contraceptive methods and factors affecting it among married women in Adigrat town, Tigray, Northern Ethiopia. Reproductive health. 2014 Dec;11(1):24.

26. Ajayi Al, Adeniyi OV, Akpan W. Use of traditional and modern contraceptives among childbearing women: findings from a mixed methods study in two southwestern Nigerian states. BMC public health. 2018 Dec;18(1):604.

27. Bwazi C, Maluwa A, Chimwaza A, Pindani M. Utilization of postpartum family planning services between six and twelve months of delivery at Ntchisi District Hospital, Malawi. Health. 2014 Jul 28;6(14):1724.

28. Federal ministry of health. routine EPI schedule. Mothers and child card. 2018.

29. Huntington D, Aplogan A. The integration of family planning and childhood immunization services in Togo. Studies in Family Planning 1994;25(3):176-83.

\section{Conclusions}

In the study area, low prevalence of modern contraceptive utilization is documented compared to the planed national figure. In the region child immunization services is one of a Promising Platform to reach lactating mothers with modern contraceptive utilization. Also our findings suggest antenatal care and postnatal care visit are the other key determinants for modern contraceptive utilization. According to our findings in a low-resource setting like Ethiopia, the health system approach to improved ANC, postnatal care and child immunization services should be intensified with more effective advice on modern contraceptive utilization to reduce unwanted pregnancy.. 